\title{
An Empirical Study on the Influence of Different Annotations on Incidental Vocabulary Learning via Reading
}

\author{
Chunxia Zhang \\ Foreign Languages School, Jingchu University of Technology, Jingmen, Hubei, 448000, \\ 568273258@qq.com
}

\begin{abstract}
Keywords: Incidental Vocabulary Learning; Reading; Chinese Annotations; English Annotations; Bilingual Annotations.
\end{abstract}

\begin{abstract}
In the process of learning a second/foreign language, vocabulary can influence learners' proficiency. Students' language performances rely heavily on their word ability. The research aims to examine 132 non-English majors in Jingchu University of Technology about their incidental vocabulary learning. Thus the researcher investigates the subjects' vocabulary levels and then carried out experiments to find out which kind of annotations, namely Chinese annotations, English annotations and bilingual (Chinese-English) annotations, is the most effective for learners to learn unfamiliar words.
\end{abstract}

\section{Introduction}

Vocabulary is the fundamental component of any language. According to Krashen, "A large vocabulary is essential for mastery of a language. Second language acquirers know this: they carry dictionaries with them, not grammar books, and regularly report that lack of vocabulary is a major problem." [1], so we can see that vocabulary is central to language and critically important to language learners [2]. In recent years, the study of second language vocabulary acquisition has become an increasingly interesting topic of discussion for researchers, teachers, curriculum designers, theorists, and others involved in second language learning [3]. Indeed, a good store of words is crucial for understanding and communication.

With regard to vocabulary acquisition, a distinction has been applied to two types of learning, i.e. 'intentional vocabulary learning' and 'incidental vocabulary learning'. Intentional vocabulary learning is a process in which students acquire vocabulary by some intentional ways, such as memorizing word list, doing vocabulary exercises. In contrast, incidental vocabulary learning is the process of acquiring vocabulary while reading or listening for comprehension rather than focusing solely on memorizing lists of words [4]. Incidental vocabulary learning first appeared in the research review of psychology in the early $20^{\text {th }}$ century. Later, based on research into how children learn vocabulary in their native language, incidental vocabulary learning was put forward by Nagy and Herman [5]. They proposed that the vast majority of vocabulary words were learned gradually through repeated exposures in various discourse contexts. Consequently, Nagy and Herman [6], argued that teachers should promote extensive reading because it could lead to greater vocabulary growth than any program of explicit instruction alone ever.

In order to improve the effect of incidental vocabulary learning, researchers turned to some activities that could help improving incidental vocabulary learning, the most commonly used activity during reading was consulting dictionary or annotations. Consulting dictionary was a useful way to deal with the unknown words, but giving the unknown words their annotations was a more effective way [7]. Cao Jia- xue and Song Jiao[8] had an experiment which shows that the traditional word list of glossing adopted by most of the current textbooks cannot facilitate students' incidental vocabulary acquisition.

\section{The Research Methods}

The Subject of Study. All of the 132 non- English majors from 3 classes in Jingchu University of Technology took part in the study. The three classes were taught by the author herself. The students 
in the three classes were in the second year of their three-year college life. Class 1, consisting of 39 students who received Chinese annotations, was designed as Experiment Group1. Class 2, consisting of 47 students who received English annotations, was designed as Experiment Group2. Class 3, consisting of 46 students who received bilingual annotations ( Chinese -English annotations), was designed as Experiment Group 3. The subjects in class1 and 2 majored in computer science, and subjects in Experiment Group3 majored in Chinese Linguistic Literature. All of the 132 subjects took the vocabulary level test. From the pretest, the subjects' vocabulary levels are the same.

Research Methods and Means. This study combines quantitative and qualitative methods, and gains data through the empirical study and interview. The empirical study includes 1) a pretest, which aims to examine the subjects' vocabulary levels. 2) a pilot study, which aims to make sure that the target words are unknown for the subjects.3) 3 vocabulary tests consisting of immediate and delayed test for each, which aim to examine whether there are any obvious progress between the three groups after vocabulary learning through different ways of annotation via3 readings.

The instrument to gather test data was Vocabulary Knowledge Scale (VKS). The VKS is particularly appropriate for this study as it distinguishes between the various stages in the developing knowledge of the target words. Paribakht, T. S. \& M. Wesche (1997) [9] demonstrated the reliability of this test through a test-retest protocol with a resulting Pearson correlation of 0.89 , indicating acceptable reliability. In order not to make any misunderstanding of the self-report, I translated the following categories into Chinese. This instrument uses a 5-point scale combining self-report and performance items to elicit self-perceived and demonstrated knowledge of specific words in written form. The scale ratings range from total unfamiliarity, through recognition of the word and some idea of its meaning, to the ability to use the word with grammatical and semantic accuracy in a sentence.

At the end of the study, an interview was conducted to know the students' attitudes towards the type of the annotations and the subjects' actual performance during the read-learn and the post-read test. It was hoped that the information from this interview would reveal more about the subjects' read-learn behavior.

\section{The Results and Analysis}

Results the First Test. Based on the descriptive of the first test, it shows the different scores of the three experiment groups in the test of the first reading. We can find from the research that in the first test, in the immediate test, the mean scores of the three experiment group are 14.4872, 14.4894, 14.4873 respectively, and in the delayed test, they are $12.6923,12.6170$ and 13.4348 . In the immediate test, the students got the very similar scores, and students receiving English annotations got litter higher scores than the other two, which means the three different annotations have nearly the same effects on the incidental vocabulary learning.

From the mean scores of the delayed test, we can know that Experiment Group3, which received bilingual annotations in the reading, got higher mean scores than Experiment Group1, which received Chinese annotations and Experiment Group2, which received English annotations. That means, bilingual annotations have better effect on the other two treatments for the word retention in the incidental vocabulary learning.

It also shows no significant difference in word test in immediate test among the three experiment groups (Sig. $=0.999>0.05)$, means that Chinese annotation, English annotations and bilingual annotations have the same effects on the incidental vocabulary learning in the English reading in the immediate test, that is the short-term retention. And it is the same with that of the delayed test (Sig. $=0.093>0.05$ ).

Results of the Second Test. In the second test, the mean scores of the three experiment groups in the immediate test are 14.6410, 14.8936 and 15.4130. In this test, Experiment Group3 got higher scores than the other two groups, which means that the bilingual annotations have the best effects on the incidental vocabulary learning in the English reading. Experiment group 2, which received English annotations, got the higher scores than Experiment Group 1. That indicates that while in the 
reading, English annotations can help the students have deeper impressions on the target words. For Experiment Group 1, which received Chinese annotations, got the lowest mean scores, indicating that Chinese annotations have the least effects on the incidental vocabulary learning in English reading.

In the delayed test, the mean scores of the three groups are 13.2564, 13.6809 and 16.5000 respectively. Compared with the immediate test, Experiment Group 3 got higher mean scores than the other two classes, which means that Experiment Group 3 have a better word retention. That is to say, bilingual annotation textual support is more beneficial to students than English annotation or Chinese annotation textual support.

The results shows that there is no significant difference among the three groups in the immediate test $($ Sig. $=0.100>0.05)$. It is clear that in this test, different annotations have little different effects on the incidental vocabulary learning in the reading. While in the delayed test, there is significant difference among the groups (Sig. $=0.000<0.05$ ), which means that in the delayed test, different annotations plays a great role in the vocabulary retention.

It also shows there is no significant mean difference among the three groups in the immediate test $($ Sig. $=0.789>0.05$; Sig. $=0.115>0.05 ;$ Sig. $=0.337>0.05)$. However, in the delayed test, there is significant mean difference between Experiment Group 1 and Experiment Group 3(Sig. $=0.000<$ 0.05). Also, there is significant difference between Experiment Group 2 and Experiment Group 3(Sig. $=0.000<0.05$ ). For Experiment Group 1 and Experiment Group 2, there is no significant difference between them (Sig. $=0.750>0.05$ ). It is clear that the text with bilingual annotations can help the subjects get better effect of incidental vocabulary learning, especially for the long-term memory.

Results of the Third Test. In the third test, the mean scores of the three groups in the immediate test respectively are 14.6410, 16.0851 and 17.0217. In the test, Experiment Group3 got much higher mean scores than Experiment Group 2 and Experiment Group 3, which indicates that the bilingual annotations have the best benefits on the incidental vocabulary learning. Experiment Group 2, which received English annotations, got the higher scores than Experiment Group 1. That means that in the reading, English annotations have better effects on the incidental vocabulary learning. Experiment Group 1 got the lowest mean scores, indicating that Chinese annotations have the least effects on the incidental vocabulary learning in English reading.

In the delayed test, the mean scores of the three classes are 13.4615, 14.2553 and 17.5000. Experiment Group 3 got the highest mean scores among the three classes, which means that Experiment Group 3 have better word retention, that means bilingual annotation textual support have the best effects to students than English annotation or Chinese annotation textual support.

It shows that there is significant difference among the three groups in the immediate test (Sig. = $0.000<0.05$ ). It can easily conclude that in this test, different annotations have different effects on the incidental vocabulary learning in the reading. In the delayed test, there is also significant difference among the groups (Sig. $=0.000<0.05$ ), which means that in the delayed test, different ways of annotations play a role in the vocabulary retention.

It is clear that in the immediate test, there is significant mean difference between Experiment Group 3 and Experiment Group 1 (Sig. $=0.000<0.05$ ), and there is also significant difference between Experiment Group 1 and Experiment Group 2 (Sig. $=0.012<0.05)$. There is no significant difference between Experiment Group 2 and Experiment Group 3 (Sig. =0.129>0.05). It indicates that the bilingual annotations have better effects on the incidental vocabulary learning than Chinese annotation. And English annotation is between the two.

In the delayed test, there is significant mean difference between Experiment Group 3 and Experiment Group 1 ( $\mathrm{Sig} .=0.000<0.05)$, but there is no significant difference between Experiment Group 1 and Experiment Group 2 (Sig.=0.356>0.05), and between Experiment Group 2 and Experiment Group 3 (Sig. $=0.000<0.05$ ). That means that bilingual annotations have better effects on the word retention than Chinese annotation. And English annotation is between the two.

Results of the Interview. Six subjects were chosen randomly from each of the experiment groups. The eighteen students from the three groups were asked for an individual interview with the 
researcher for about 10 minutes, during which the researcher took notes of important points at the same time. The interview consists of following questions: Compared with reading without annotations, do you think reading with annotations can help you learn more words? Which kind of annotations is the most effective?

To most subjects, the merits of annotations are apparent: they are direct and concrete, and require no extra cognitive efforts to figure out the meanings, ensuring the fluency of reading process, the annotations inserted in the text draw relatively more attention of a learner to the associated words. All of them thought that the annotations in the reading materials can help them a lot in the reading comprehension and vocabulary learning. The findings of the present study conducted by the writer gave more evidence to the benefit of the use of annotation and this can surely do help in the application of the annotation support into practical use.

As for different ways of annotations, the interviewees under Chinese annotation treatment would pay attention to the meaning of the annotated words, but they seldom paid much attention to the characteristics of the words. The interviewees under English annotation treatment would treat the annotations in different ways: some students thought English annotations were useful, they would pay special attention to the usage of the words, but the others thought English annotations sometimes would take up a lot of time during the reading. And the interviewees under bilingual treatment all agreed that when they encountered the annotations, they would first focus on the Chinese annotations, then the English annotations.

\section{Discussions}

The students' perceptions on annotations can be explained by the theory of Depth of Processing Hypothesis, in which Craik and Lockhart [10] argued that there were different levels or depths of information processing and the chance that some piece of new information would be stored in long-term memory was not determined by the length of time that was held in short-term memory but rather by the shallowness or depth with which it was previously processed. It was natural that for the students, the more time and effort they spared on the information of the words, the best they would keep the information in their mind.

For the subjects with the same vocabulary levels, those under bilingual annotations outperformed those under other two treatments. And for Chinese annotations and English annotations, those under English annotations outperformed those under Chinese annotation treatment. Possibly, it is because English annotations, though it was not so clear and comprehensible as Chinese annotations, when compared with the Chinese annotations, they are more beneficial for the students to put them in the memory.

\section{The Suggestions}

To Form Effective Vocabulary Learning Strategies. The bilingual (Chinese-English) annotation is more beneficial to Chinese three-year college students' vocabulary learning via reading. So, while learning vocabulary, the students can make effort to give Chinese-English annotation in the reading in order to acquire new words.

To Make Better Choice for Reading Materials. The result of the research can benefit English teachers, English learners and extracurricular English material editors. Since enlarging vocabulary size is the major task in the process of English learning, the teachers' task is to help the students learn more vocabulary. Therefore, teachers can choose those materials with different annotations to difficult words and distribute them to their students for classroom or extracurricular reading. Thus, it can become a reference for extracurricular reading material editors to design the annotations in a better way and make their materials more helpful to students. 


\section{Conclusion}

A good store of words is crucial for understanding and communication. Good language performers are usually good vocabulary acquirers. Students' language performance relies heavily on their word ability. An effective way of acquiring vocabulary is through incidental vocabulary learning via extensive reading. The bilingual annotations have better effect on the learners than the other two annotations, that is, Chinese annotations and English annotations. Subjects with different vocabulary levels have different attitudes towards different annotations, that is to say, among the three different annotation ways, Chinese annotations only give the Chinese meaning of the target words, and English annotations can draw more attention and effort of the subjects', thus the subjects have a deeper impression on the target words, bilingual annotations, which have the two advantages, can help the subjects the most.

\section{References}

[1] Krashen, S. D, We acquire vocabulary and spelling by reading: Additional evidence for the input hypothesis, J. the Modern Language Journal. 73(1989), 440-464.

[2] Manchun Dai, Theoretical bases of second language vocabulary acquisition, J. Foreign Language Teaching and Research. 32(2000)138-144. (In Chinese)

[3] Coady, J, Second vocabulary acquisition: A synthesis of the research, In J. Coady \& T. Huckin (eds.), Second language vocabulary acquisition. New York: Cambridge University Press. 1997a, pp 273-290.

[4] Hulstijn, J. H., M. Hollander.\&T. Greidanus, Incidental vocabulary learning by advanced foreign language students: The influence of marginal glosses, dictionary use, and reoccurrence of unknown words, J. Modern Language Journal. 80 (1996) 327-339.

[5] Nagy, W, Herman, P. Incidental vs. instructional approaches to increasing reading vocabulary, Educational Perspectives. 1985 (23): 16- 21.

[6] Nagy, W. E., P. Hetman, Breadth and depth of vocabulary knowledge: Implications for acquisition and instruction, In M. McKeown and M. Curtis (Eds.). The nature of vocabulary acquisition. Mahwah, NJ: Lawrence Erbium. 1987: 19-35.

[7] Hulstijn, J. H., M. Hollander.\&T. Greidanus, Incidental vocabulary learning by advanced foreign language students: The influence of marginal glosses, dictionary use, and reoccurrence of unknown words, J. Modern Language Journal.1996

[8] Jia-xue Cao, Jiao Song, Effects of Three Ways of Glossing on Incidental Vocabulary Acquisition, J. Forengn Language Research. 176(2014) 117-120. (In Chinese)

[9] Paribakht, T. S., M. Wesche. Enhancing vocabulary acquisition through reading: A hierarchy of text-related exercise types, Canadian Modern Language Review. 12(1996) 15-28.

[10]Craik, F. I., R. S. Lockhart. Levels of processing: A framework for memory research, J. Journal of Verbal Learning and Verbal Behavior, (1972) 89-106 